\title{
LEÇON DES FRONTIÈRES FANTÔMES : LES TRACES DU PASSÉ NOUS VIENNENT (AUSSI) DU FUTUR
}

\author{
Béatrice von Hirschhausen
}

Belin | «L’Espace géographique »

2017/2 Tome 46 | pages 97 à 105

ISSN 0046-2497

ISBN 9782410008654

Article disponible en ligne à l'adresse :

https://www.cairn.info/revue-espace-geographique-2017-2-page-97.htm

Distribution électronique Cairn.info pour Belin.

(C) Belin. Tous droits réservés pour tous pays.

La reproduction ou représentation de cet article, notamment par photocopie, n'est autorisée que dans les limites des conditions générales d'utilisation du site ou, le cas échéant, des conditions générales de la licence souscrite par votre établissement. Toute autre reproduction ou représentation, en tout ou partie, sous quelque forme et de quelque manière que ce soit, est interdite sauf accord préalable et écrit de l'éditeur, en dehors des cas prévus par la législation en vigueur en France. Il est précisé que son stockage dans une base de données est également interdit. 


\title{
Frontières fantômes
}

\section{$2017-2$}

p. 97-105

\section{Leçon des frontières fantômes: les traces du passé nous viennent (aussi) du futur}

\author{
Béatrice VON HIRSCHHAUSEN \\ CNRS-UMR 8504 Géographie-cités \\ 13 rue du Four \\ F-75006 Paris \\ hb@cmb.hu-berlin.de
}

Und ne métaphore dans les sciences est créative, écrit l'épistémologue et linguiste Peter Finke, "quand elle donne accès à une perspective inédite sur un paysage complexe que l'on ne saurait saisir en totalité mais que le nouveau point de vue permet de considérer de manière plus complète et moins déformée $»^{1}$ (Finke, 2003, p. 55).

C'est avec l'intuition que l'analogie avec le fantôme offrait une nouvelle manière de questionner les longues durées géographiques que j'avais formé en 2009 le terme nouveau de "Phantomgrenzen» en allemand ("frontières fantômes») en vue d'un projet interdisciplinaire qui devait réunir, en Allemagne, des spécialistes d'Europe centrale et orientale, sur la question des différences géoculturelles. Le collectif a réuni une vingtaine de chercheurs, entre 2010 et $2017^{2}$, autour des phénomènes de rémanence de territorialités impériales révolues dans les espaces contemporains, en partant du repérage de leurs traces sur certaines cartes thématiques, dans des paysages ou dans des représentations communes. Nous avons nommé ces traces «frontières fantômes» par analogie avec les apparitions, hésitant entre corporéité et fantasme, de défunts ou de vaisseaux disparus. L'idée de frontières fantômes (nous parlons aussi dans certains cas de "spatialités fantômes", de "territorialités fantômes» ou de "géographies fantômes») joue de cette double nature corporelle et fantasmatique; elle joue aussi d'une métaphore secondaire, celle des douleurs fantômes ressenties dans un membre amputé. Du fantôme, les rémanences spatiales étudiées tiennent le même caractère imprévisible: elles peuvent apparaître sur des cartes à l'occasion d'un rendez-vous électoral, par exemple, mais rester invisibles sur d'autres cartes. Les participants au projet, principalement des historiens et des géographes, se sont saisis de la métaphore pour explorer une nouvelle perspective sur les longues durées géoculturelles dans cette partie d'Europe où les États-nations ont été découpés et recomposés dans des morceaux d'empires (fig. 1).

1. «Eine kreative Metapher ist wie die Gewinnung eines veränderten Aussichtspunktes auf eine komplexe Landschaft, die man nie vollkommen überblicken kann, die aber vom neuen Aussichtspunkt aus allem Anschein nach besser, vollständiger, weniger verzerrt gesehen werden kann». Traduit par nous.

2. Le projet "Phantomgrenzen in Ostmitteleuropa » ("les frontières fantômes en Europe centrale et orientale ") a été développé dans le cadre d'un réseau de recherche financé entre 2011 et 2017 par le ministère fédéral allemand de l'Enseignement et de la Recherche.

Voir www.phantomgrenzen.eu 


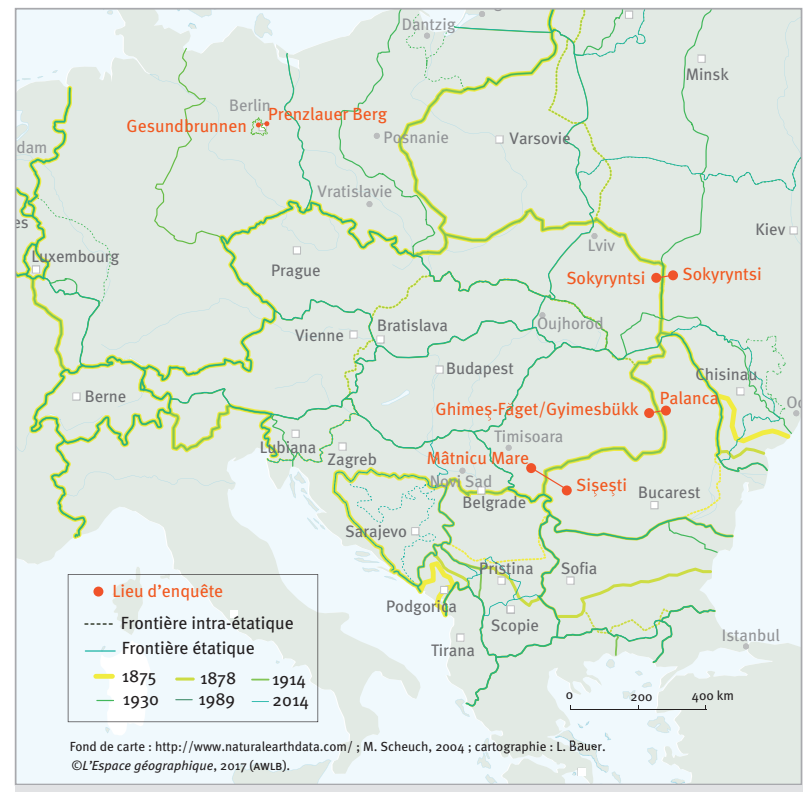

Fig. 1/ Localisation des études de cas sur le palimpseste des frontières

On a représenté ici de manière cumulative l'ensemble des frontières politiques qui ont été tracées depuis 1875 sur l'espace cartographié. Cette carte, inspirée de celle que Michel Foucher avait publiée dans Fragment d'Europe (1995), n'a pas vocation à être lue de manière analytique : on peinerait à y repérer les différentes configurations historiques. Elle a, en revanche, l'intérêt de donner à voir la densité des redécoupages frontaliers qui ont scandé la décomposition des empires et la recomposition des États-nations au cours des derniers 150 ans en Europe centrale et orientale. Elle laisse deviner l'intensité des expériences frontalières et l'actualité, pour les sociétés locales en cet « entre-deux » géopolitique (Rey, 1995), de la question des fantômes territoriaux.
Une hypothèse initiale a fondé le travail commun: nous avons considéré que les fantômes de territorialités impériales, dans les géographies contemporaines, étaient le fait (à la fois individuel et collectif) de pratiques ordinaires quotidiennes et que l'on pouvait, à partir de la compréhension de ces pratiques vernaculaires, renouveler la question du rapport espaces/histoire/culture dans une plus longue durée. De cette hypothèse sont issus les deux choix méthodologiques qui ont piloté les travaux du collectif. Le premier choix a été celui d'une approche de type inductif, construite dans un va-etvient entre une panoplie d'études de cas conduites à un niveau local et l'élaboration conceptuelle; ce premier choix a permis de se dégager d'un débat général sur la pertinence des aires culturelles, qui reposait jusqu'alors sur des approches surplombantes des différences géoculturelles. L'idée consistait donc à aller observer au plus près la production locale de discontinuités culturelles dans les pratiques et/ou les représentations et de chercher à élaborer une proposition d'ambition théorique, dans une confrontation serrée avec ces matériaux empiriques. Le second choix méthodologique a été d'interroger la production vernaculaire des frontières fantômes à partir de trois entrées que nous voulions considérer conjointement: celle des acteurs, de leurs agirs et de leurs choix, celle de leur expérience historiquement et géographiquement située, et enfin celle de leurs imaginaires, de leurs croyances et de leurs attentes. Nous choisissions ici de récuser l'opposition qui depuis une vingtaine d'année organise en deux postures antinomiques le champ d'étude des aires culturelles entre, d'un côté, les analyses des discours hégémoniques dont les études postcoloniales se sont fait la spécialité et, de l'autre, les analyses des structures de longue durée des approches classiques. En plaçant au centre de l'analyse une approche microgéographique des agirs individuels et collectifs dans leur double relation à l'espace structuré-structurant de l'expérience et aux horizons d'imaginaire et de croyance, nous avons vu le moyen d'échapper à cette antinomie. En concevant les frontières fantômes comme conjointement produites, expérimentées et imaginées par les acteurs locaux, nous avons cherché à réarticuler sur un mode original le présent de la production concrète des espaces à la longue durée de l'accumulation des expériences collectives et aux projections dans des futurs imaginés.

Les premiers résultats de ce travail ont été publiés pour un lectorat germanophone dans un ouvrage programmatique édité en 2015 (Hirschhausen et al., 2015) précédé et suivi de plusieurs publications développant les études de cas (Löwis, 2015a et b; Aldenhoff-Hübinger et al., 2016; Esch, 2016; Tomic, 2016; Esch, Hirschhausen, 2017; Müller, Struve, 2017). 


\section{Étude dle quatre frontières fantômes dans l'Europe postsocialiste}

L'objet du présent dossierl est de poursuivre la recherche et d'en transférer certaines propositions dans la sphère francophone, ici à partir de quatre études conduites sur des frontières fantômes apparues au cours de la période postsocialiste. Trois des contributions portent sur les fantômes des frontières orientales des territoires Habsbourg dont l'établissement ou la rigidification date de la fin du XVIII ${ }^{\mathrm{e}}$ siècle et la remise en cause de la fin de la Première Guerre mondiale; la quatrième porte sur le fantôme de la frontière du mur de Berlin, dont l'existence historique fut à la fois plus récente et plus brève (1961-1989).

Le dossier s'ouvre sur un article de Béatrice von Hirschhausen ${ }^{3}$ qui permet de situer l'intérêt heuristique des frontières fantômes pour les débats sur le rapport espace/histoire/culture, débats qui ont connu en Allemagne une résonance particulièrement importante. Partant d'une étude de cas qui voit l'apparition d'une discontinuité dans la géographie de l'équipement domestique dans les campagnes de Roumanie, l'article débouche sur deux thèses qui prolongent et approfondissent les propositions collectives du projet. L'article montre premièrement que la frontière fantôme, apparue sur la carte de la modernisation des villages, n'est pas la manifestation d'une différence immuable ou immémoriale qui distinguerait une Europe centrale plus apte à la modernité d'une Europe balkanique qui y serait rétive, mais qu'elle peut être analysée, dans son historicité même, comme le résultat des représentations géographiquement différenciées que se font les habitants des défis postsocialistes et de leurs aptitudes à les relever. Autrement dit, les différences entre les ressources sociales, matérielles ou culturelles mobilisables de part et d'autre par les villageois pour accéder à la modernité souhaitée ne sauraient se comprendre sans prendre également en compte les représentations que les villageois s'en font. L'asymétrie des modernisations entre l'ouest et l'est des Carpates peut être alors lue moins comme une reconduction des écarts de développement hérités du passé que comme une sorte d'actualisation à rebours, par les acteurs villageois, des futurs qu'ils imaginent possibles ou probables pour leur région. L'article soutient deuxièmement la thèse d'une puissance performative des cartes mentales, produites à une toute autre échelle par les savoirs institués et qui structurent nos imaginaires du monde comme de l'Europe en attribuant des qualités, des valeurs et des destinées aux lieux et aux territoires. Les futurs régionaux que les acteurs locaux tiennent pour plausibles et qui guident leurs choix, sont construits et établis par une «géographie de l'Histoire" (Grataloup, 2011) qui organise spatialement le grand récit du progrès et livre implicitement des pronostics d'avenir pour les différentes parties du monde. Sa puissance performative est d'autant plus efficace qu'elle est le plus souvent perçue par les acteurs locaux comme naturelle ou évidente. Elle se condense localement en toute une série de qualifications associées au lieu («ici, c'est comme cela», «ici, on fait ainsi») qui fonctionnent autant comme des constats que comme des injonctions à s'y conformer.

Contrairement à ce que pourrait laisser croire la teneur programmatique de ce premier article, ceux qui suivent dans le dossier n'ont pas été écrits à sa suite mais en parallèle; ils ne s'emparent donc pas complètement de son schéma interprétatif et proposent une analyse compréhensive de «leurs» frontières fantômes respectives à partir $\mathrm{du}$ dispositif plus ouvert mis en place en amont pour l'ensemble du projet. Leurs auteurs ont été impliqués à des degrés très divers au sein du réseau de recherche et ils ont en commun de s'intéresser, comme le premier article du dossier, à la recomposition
3. Hirschhausen B. von (2017). " De l'intérêt heuristique du concept de "fantôme géographique" pour penser les régionalisations culturelles ". L'Espace géographique, t. 46, $\mathrm{n}^{\circ} 2$, p. 106-125. 
4. Löwis S. von (2017). "Frontières fantômes et ambivalence des espaces d'identification en Ukraine ". L'Espace géographique, t. 46, $\mathrm{n}^{\circ} 2$, p. 141-157. des espaces d'identification, issue de l'effondrement du système socialiste. Les articles de Sabine von Löwis et de Gabor Szalkai s'intéressent à de nouvelles sémiotisations de l'espace qui ont accompagné la sortie du système socialiste et l'abandon de son grand récit prophétique: la première dans des campagnes ukrainiennes, le second dans les Carpates roumaines, dans une vallée où se côtoient des populations de langue roumaine et de langue magyare.

Pour Sabine von Löwis ${ }^{4}$, il s'agit de produire une interprétation de la frontière fantôme observée de manière récurrente sur les cartes électorales des dernières décennies dans l'ouest de l'Ukraine. Dans deux villages jumeaux situés sur la Zbroutch, sur l'ancienne frontière impériale qui sépara l'Empire Habsbourg de l'Empire russe, et qui aujourd'hui organise une partie de la géographie des votes "pro-russe " ou "pro-européen " des cartes électorales, elle y interroge les modalités concrètes par lesquelles se réarticulent, depuis l'effondrement soviétique et l'indépendance de l'Ukraine en 1991, les espaces d'identification et les affiliations politiques. Elle examine pour cela les différents monuments qui ponctuent les deux espaces villageois et le sens que les habitants leur donnent. Un premier tour de repérage pourrait d'abord laisser croire à l'existence de deux systèmes de référence historiques opposés. Les événements et figures célébrées de part et d'autres ne se recoupent pas : à l'est, c'est la mémoire du communisme, de ses figures tutélaires comme de ses crimes, qui est mise en monument, à l'ouest c'est celle du nationalisme ukrainien cohabitant avec celle des tombes de ses victimes polonaises. Mais l'analyse des témoignages des habitants révèle des affiliations labiles et ambiguës, loin des oppositions tranchées. Elle montre que la construction, dans les années 2000, de deux nouveaux monuments l'un, à l'est, pour commémorer la famine de 1932-1933 dans l'Ukraine soviétisée et l'autre, à l'ouest, pour célébrer les combattants de l'armée insurrectionnelle ukrainienne des années 1940, correspondent à deux transcriptions locales d'une même mémoire culturelle nationale ukrainienne promue par Kiev depuis vingt-cinq ans et ajustées aux deux expériences historiques séparées des villageois. Mais le récit national qui porte cette mémoire culturelle ne coïncide que malaisément avec les mémoires communicatives locales, transmises dans les entre-soi villageois et familiaux, et tissées d'ambivalences et de contradictions. S. von Löwis repère des rapports locaux au passé, rétifs aux embrigadements et aux lectures manichéennes de l'histoire. Au terme de son analyse fine des représentations de part et d'autre de la petite rivière, elle ne saurait conclure à l'existence de deux communautés distinctes et territorialisées. La frontière, quand on l'observe à cette échelle, est plus que jamais fantomatique. Elle ne tient pas du modèle de la "frontière invisible» (François, 1993) qui organise et régule les rapports entre deux communautés. Ce n'est pas un seuil.

Resterait alors à comprendre ce qui fait la constance de la discontinuité repérable sur les cartes électorales des grands rendez-vous politiques des dernières décennies. S. von Löwis suggère une interprétation qui articule les choix du présent aux représentations du passé sur un mode original et indirect. Contrairement à ce que laissent entendre les analyses qui glissent trop vite de la carte électorale à la carte historique, l'étude de S. von Löwis conteste l'existence d'un lien mécanique qui aurait naturellement porté les sociétés villageoises posthabsbourgeoises vers des partis plus libéraux et pro-européens, par la transmission d'habitus politiques ou de normes sociales qui seraient propres aux communautés de l'ouest galicien enraciné dans son passé Habsbourg et qui les différencieraient de leur voisines de Podolie et de leur passé impérial russe. Elle montre que c'est bien davantage du projet national 
ukrainien formulé dans les années 1990 et 2000 qu'ont émergé les différences entre les modalités locales de célébration de la Nation. Le choix, fait depuis l'Indépendance en 1991, de recomposer une identité nationale non pas sur un projet politique ou un projet de société tourné vers le futur mais sur des lieux de mémoires du passé (grande famine de 1932, armée nationaliste ukrainienne) n'est, dans l'Europe actuelle, certes ni propre à l'Ukraine ni original. Mais il a pour effet, dans une nation partagée, il y a encore trois générations, entre des entités étatiques distinctes, de "spatialiser les mémoires" au sens où c'est l'Ouest ukrainien qui se retrouve confondu avec l'histoire du nationalisme et de son armée insurrectionnelle, tandis que les régions d'Ukraine centrale sont associées à la mémoire de l'expérience soviétique. À partir du schéma dynamique d'analyse que nous avons élaboré selon les catégories de «champ d'expérience» et d'«horizon attente» forgées par Reinhardt Koselleck et qui interprète les frontières fantômes à l'intérieur d'un arc de tension tenant ensemble l'expérience géographiquement située et "saturée de réalité" des acteurs et leurs imaginaires du futur (Koselleck, 1990), on peut faire l'hypothèse que les affiliations partisanes et les futurs "pro-russes» ou "pro-européens» qui les définissent tendent à s'établir en fonction des différents territoires d'identification du nouveau discours national. La «spatialisation des mémoires» qui a conduit à associer la mémoire de différents moments historiques à telle ou telle portion du territoire national pourrait avoir débouché, selon cette analyse, sur une forme de spatialisation des affiliations partisanes et de leurs visions du futur : les régions occidentales qui se retrouvent aujourd'hui identifiées, dans le nouveau discours mémoriel de la nation Ukrainienne, avec la mémoire anti-soviétique du nationalisme ukrainien ne sauraient voter "pro-russe» à la différence des régions de l'est du Zbroutch qui sont identifiées avec l'expérience soviétique et à sa résistance à l'envahisseur fasciste. Le fantôme de la frontière impériale apparu sur les cartes électorales et la spatialisation des affiliations partisanes qu'il révèle ne sauraient alors se comprendre comme la reproduction dans la durée d'anciens habitus politiques, mais plutôt comme l'expression de manières actuelles et situées d'interpréter les trajectoires historiques. Les options électorales locales s'éclairent alors au prisme des différentes manières locales de se situer entre Est et Ouest et de reconstruire, dans le contexte particulier de l'Ukraine postsoviétique, les relations entre passé, présent et futur.

G. Szalkai 5 s'est attelé de son côté à l'analyse d'une spectaculaire entreprise de sémiotisation de l'espace, conduite par des acteurs locaux et transnationaux dans une haute vallée des Carpates au centre de la Roumanie, sur un autre segment de l'ancienne frontière impériale de la double monarchie Habsbourg. Jusqu'en 1921 et le traité de Trianon, on était ici dans le royaume de Hongrie. La région a ensuite été incorporée à la Roumanie, conservant toutefois des villages à majorité magyarophone. Il décrit comment certains acteurs, locaux et budapestois, ont réveillé la mémoire de la frontière au passage de l'ancien poste douanier en y créant année après année, à partir de 1996, tout une série de monuments commémorant l'Empire et l'histoire hongroise de ces confins. L'extraordinaire succès d'un pèlerinage annuel lancé en 2008 sur ce site et la hausse de la fréquentation touristique de la commune par une clientèle hongroise, l'ont érigé en véritable haut lieu de la mémoire hongroise, l'incorporant en quelques années dans le grand récit national. G. Szalkai décrit aussi comment les villages voisins roumanophones sur le versant moldave du massif ont à leur tour mobilisé leurs propres figures tutélaires pour célébrer non pas la frontière mais son abolition en 1921. Au-delà de cette concurrence des mémoires, ce qui se joue ici
5. Szalkai G. (2017). "Quand les "fantômes historiques" sont convoqués: invention de hauts lieux et réification des identités territoriales dans les Carpates roumaines ". L'Espace géographique, t. $46, \mathrm{n}^{\circ} 2$, p. 126-140. 
6. Hocquet M., Garrido C., Hirchhausen B. von (2017) «Berlin par-delà les ruptures. Vivre, raconter et produire les matières de la ville ". L'Espace géographique, t. 46, nº 2 , p. 158-173. avec la convocation du fantôme frontalier est une forme de "dramatisation» (pour parler comme Edward Said) de la différence culturelle, renvoyant les villages roumains à leur altérité "balkanique» et distinguant la culture hongroise par son appartenance à la monarchie Habsbourg et au legs centre-européen. Le processus met à mal les hybridations linguistiques et identitaires des populations locales et tend à segmenter ces dernières en communautés ethnolinguistiques. Il ne saurait prétendre à l'innocence que son emballage touristique voudrait laisser accroire: la situation locale décrite par G. Szalkai permet d'éclairer ce qui se passe quand certains "entrepreneurs de mémoire» choisissent de "faire tourner les tables» en quelque sorte et de convoquer sciemment le fantôme d'une frontière. Ce qui se joue dans la petite région du Ghimeş/Gyimes tient de la fabrique d'un haut lieu tel que l'a défini Bernard Debarbieux (1995). Il fonctionne désormais, pour une Nation hongroise nostalgique, comme un "lieu de condensation» symbolique du territoire partagé. Il signifie et exalte l'ancien royaume de Hongrie dont il reconstitue symboliquement la continuité en l'émancipant des contingences historiques de son démembrement. Il le donne à voir et le donne à vivre en quelque sorte en permettant de ne pas le laisser «cantonné dans l'immatériel et l'imagination. Il [le] rend crédible et devient lui-même objet de cette croyance». (Debarbieux, 1995, p. 108). Si l'on suit les propositions de B. Debarbieux jusqu'au bout, ce nouveau haut lieu apparaît comme une pièce maîtresse de la fabrique d'un territoire qui conteste l'ordre spatial et temporel des frontières actuelles. Ici le fantôme participe d'une nouvelle construction de la réalité.

Le lecteur attentif des articles de S. von Löwis et G. Salkai repèrera sans doute des différences de sensibilité entre les deux textes, entre leurs manières respectives de saisir le rapport espace/histoire/culture ici en jeu. C'est bien sûr affaire de différences personnelles entre les deux auteurs et entre leurs curiosités intellectuelles respectives. Nous y repérons aussi l'empreinte des champs nationaux au sein desquels ils situent leurs travaux et des enjeux politiques qui les traversent. L'attention accordée de manière prioritaire par $S$. von Löwis aux ambivalences et aux modalités hybrides de construction des affiliations identitaires des villageois qu'elle étudie est à resituer dans un champ de la géographie allemande actuelle particulièrement soucieuse d'éviter tout risque de naturalisation, même indirecte, des identités régionales; cette préoccupation est ici d'autant plus essentielle que les analyses communes du conflit ukrainien, véhiculées par ses acteurs politiques comme par les media, ont cherché à articuler systématiquement les identités politiques à des entités territoriales historiques plus ou moins figées. Ce souci n'est en revanche pas celui de G. Szalkai, quand il analyse l'actualité touristique et symbolique de la "frontière de mille ans", que la grande majorité de ses compatriotes hongrois considère comme naturelle. L'enjeu pour lui est moins de conduire la critique de sa "mémorialisation » que d'en décrire et éclairer la vigueur.

Le dernier article ${ }^{6}$ porte sur Berlin et la frontière du Mur. Il mobilise les matériaux empiriques de ses trois auteures pour questionner la manière dont le passé du partage s'actualise dans l'espace urbain. Le concept de frontière fantôme est ici mobilisé pour interroger une production vernaculaire des espaces à la fois «habitée et habillée d'histoire». Les auteures cherchent à comprendre comment articuler les trois niveaux de construction identifiés au sein du réseau de recherche pour concevoir les spatialités fantômes : le niveau de la production sociale et matérielle de la ville, le niveau des imaginaires de l'espace urbain et le niveau de l'expérience que les acteurs en ont. À partir de différents matériaux empiriques, produits à l'échelle micro de deux 
études ethnographiques et historiques et à l'échelle de la cartographie électorale, elles explorent comment les acteurs, qu'ils soient habitants, électeurs, citoyens engagés ou passants, pratiquent, imaginent et produisent la ville. L'analyse permet de sonder comment des manières de dire, de vivre et de produire l'urbain peuvent rééditer des bribes du passé et du partage de la Guerre froide. Cette lecture va à l'encontre de l'image généralement mobilisée pour éclairer les rémanences et les longues durées spatiales d'un passé "sédimenté» en couches successives. Elle met plutôt en lumière des processus actifs, créatifs, de construction de la ville entre la puissance instituante des héritages spatiaux d'une part, et d'autre part, des imaginaires qui donnent sens et valeur à ces legs du passé.

\section{Frontières fantômes, régimes d'historicité et production des espaces ordinaires}

Que nous disent ces quatre fantômes de la géographie postsocialiste de l'Europe et, au-delà, de la production des espaces? Ils sont apparus sur nos cartes de l'Europe centrale et orientale au cours des premières décennies après l'effondrement de l'ordre socialiste: pour les uns avec les premières élections libres, pour d'autres avec la liberté de voyager et de s'exprimer ou avec les processus de modernisation des campagnes. On pourrait les interpréter comme la mise à jour, du seul fait de l'apparition d'indicateurs pour les mesurer, de différences spatiales préexistantes, incorporées dans les institutions ou dans les normes et pratiques sociales, mais demeurées invisibles sous le socialisme. Le lecteur l'aura compris, ce n'est pas le parti que nous avons pris.

Violette Rey, dans son analyse des longues durées géographiques de l'espace centre et est-européen, avait montré l'intérêt de cet espace "entre-deux», balloté par les déplacements de frontières et les basculements successifs des hégémonies extérieures (Rey, 1995). Son travail pour capter les modalités spatiales et temporelles propres à la géographie de la région a mis l'accent sur les fragmentations paysagères et les particularités des expériences historiques qui y sont associées. «Les paysages, fragments de temps piégés, [...] expriment dans l'espace la particularité du rapport que les sociétés entretiennent avec le temps» écrivait-elle dans la Géographie universelle à propos de "l'Autre Europe» (Rey et al., 1996, p. 10). Cette approche faisant place à la signification existentielle pour les habitants de leur position géographique pour éclairer conjointement leurs manières particulières de se comprendre dans l'histoire et de produire l'espace ordinaire, a ouvert une voie de recherche que nous continuons d'explorer, ici à un niveau microgéographique.

Dans les études de cas que nous proposons dans ce dossier, on voit se dessiner en filigrane la variété des interprétations que les sociétés locales développent à un moment donné de leur propre trajectoire historique ou de celles de sociétés voisines: que ce soit à partir de l'analyse des monuments qui ponctuent le territoire, lui donnent sens et le situent dans une narration nationale, ou à partir de l'analyse des stratégies de modernisation domestique ou des modalités de rénovation urbaine, les quatre terrains ici étudiés exploitent des moments de redéfinition du rapport à l'histoire, à la fois comme récit et comme expérience. Ce rapport a trait à ce que Francois Hartog (2003) a nommé - en se fondant sur Reinhardt Koselleck - les «régimes d'historicité" pour désigner dans leur pluralité les modalités particulières par lesquelles les sociétés se pensent dans l'histoire et se vivent dans le temps. Or ce qui est particulièrement intéressant ici, c'est que les 
frontières fantômes, en en dessinant les variations spatiales, révèlent des discontinuités géographiques entre différents modes d'articulation du passé, du présent et du futur. Comme si l'expérience vécue conjointement par les sociétés centre-est-européennes, de l'effondrement du messianisme communiste et de la refonte complète de l'ordre géopolitique de l'Europe, mettait à jour ce qui noue intimement position géographique et régime d'historicité. À bien des égards, l'environnement géohistorique du postsocialisme centre et est-européen, permet d'examiner plus avant ce qui articule les régimes d'historicité aux contextes situés de leur déploiement.

C'est ici que l'on peut situer la portée plus générale d'une recherche conduite dans une partie de l'Europe mais dont nous pensons qu'elle peut être utile à la compréhension d'autres terrains de par le monde. Les études empiriques que nous produisons ici montrent au moins deux choses. Elles montrent que la manière dont les sociétés locales se pensent dans l'histoire et se vivent dans le temps est tributaire de la manière dont elles se pensent dans l'espace géographique et dont elles vivent leur territoire. Elles montrent aussi qu'en retour la production vernaculaire des espaces et des territoires ne se fait pas (et ne saurait se comprendre) dans un temps unifié du monde ou dans une grande narration globale, mais qu'elle se fait sous des régimes d'historicité particuliers et situés, tissés d'expériences et d'imaginaires du futur.

\section{Références}

Aldenhoff-HüBinger R., Gousseff C., SerRier T. (dir.)(2016). Europa vertikal. Zur Ost-West-Gliederung im 19. und 20. Jahrhundert. Göttingen: Wallstein-Verlag, $230 \mathrm{p}$.

Debarbieux B. (1995). « Le lieu, le territoire et trois figures de rhétorique ». L'Espace géographique, t. 24, no 2, p. 97-112.

EscH M.G. (2016). Die Stadt als Spielfeld. Raumbegriffe, Raumnutzungen, Raumdeutungen polnischer Hooligans. Göttingen: Wallstein-Verlag, $120 \mathrm{p}$.

Esch M.G., HirschHausen B. von (dir.)(2017). Wahrnehmen, erfahren, gestalten. Phantomgrenzen und soziale Raumproduktion. Göttingen: Wallstein-Verlag, $180 \mathrm{p}$.

Finke P. (2003). «Misteln, Wälder und Frösche: Über Metaphern in der Wissenschaft ». Metaphorik.de, $\mathrm{n}^{\circ}$ 4, p. 45-65. http://www.metaphorik.de/sites/www.metaphorik.de/files/journal-pdf/04_2003_finke.pdf

FOUCHER M. (dir.)(1995). Fragments d'Europe. Atlas de l'Europe médiane et orientale. Paris: Fayard, $328 \mathrm{p}$.

Françols É. (1993). Protestants et catholiques en Allemagne. Identités et pluralisme. Augsbourg 1648-1806. Paris: Albin Michel, coll. «L'Évolution de l'humanité», 392 p.

Grataloup C. (2011). Faut-il penser autrement l'histoire du monde? Paris: Armand Collin, coll. «Éléments de réponse» $216 \mathrm{p}$.

Hartog F. (2003). Les Régimes d'historicité : présentisme et expériences du temps. Paris: Éditions du Seuil, coll. «La Librairie du xxı siècle», 272 p.

Hirschhausen B. von, Grandits H., Kraft C., Müller D., Serrier T. (dir.)(2015). Phantomgrenzen: Räume und Akteure in der Zeit neu denken. Göttingen : Wallstein-Verlag, $224 \mathrm{p}$.

Koselleck R. (1990). Le Futur passé. Contribution à la sémantique des temps historiques. Paris: Éditions de l'École des hautes études en sciences sociales, $334 \mathrm{p}$.

LöwIS S. von (dir.)(2015a). «Phantom borders in the political geography of East Central Europe: An introduction». Erdkunde, vol. 69, nº 2, p. 99-106. 
LöwIS S. von (dir.)(2015b). «Einführung - Zur Zeitlichkeit räumlicher Konstrukte: Grenzen und Regionen in Vergangenheit und Gegenwart». Europa Regional, n³ 3-4, p. 83-89.

MülleR M.G., StRuVE K. (dir.)(2017). Fragmentierte Republik? Das politische Erbe der Teilungszeit in Polen 1918-1939. Göttingen: Wallstein-Verlag, $200 \mathrm{p}$.

ReY V. (1995). «L'Europe centre orientale, un “entre-deux” ». In Bailly A., FerRAS R., Pumain D. (dir.), Encyclopédie de géographie. Paris: Economica, p. 813-825.

Rey V., Billaut M., Daniel O., Roux M. (1996). «Europes orientales». In Brunet R., Rey V. (dir.), Géographie Universelle. T. 10: Europes orientales, Russie, Asie centrale. Paris: Belin, p. 7-206.

Томіс́ Đ. (2016). Phantomgrenzen und regionale Autonomie im postsozialistischen Südosteuropa. Die Vojvodina und das Banat im Vergleich. Göttingen: Wallstein-Verlag, $357 \mathrm{p}$. 